See discussions, stats, and author profiles for this publication at: https://www.researchgate.net/publication/312481289

\title{
Photodynamic Inactivation of Cariogenic Pathogens Using Curcumin as Photosensitizer
}

Article in Photomedicine and Laser Surgery · January 2017

DOI: 10.1089/pho.2016.4156

CITATIONS

5

8 authors, including:

Natália Costa Araújo

Universidade de Pernambuco

30 PUBLICATIONS 218 CITATIONS

SEE PROFILE

A) Carla R Fontana

São Paulo State University

59 PUBLICATIONS 1,420 CITATIONS

SEE PROFILE

Some of the authors of this publication are also working on these related projects:

Project Developed a new technological setup design with ozone process, UV process View project

Project Bell's Palsy View project
READS

119

Vanda Sanderana Macêdo Carneiro

Universidade de Pernambuco

22 PUBLICATIONS 41 CITATIONS

SEE PROFILE 


\title{
Photodynamic Inactivation of Cariogenic Pathogens Using Curcumin as Photosensitizer
}

\author{
Natália Costa Araújo, PhD, Rebeca Ferraz de Menezes, MD, 2,3 \\ Vanda Sanderana Macêdo Carneiro, MD, ${ }^{1,7}$ Alexandrino Pereira dos Santos-Neto, MD, \\ Carla Raquel Fontana, $\mathrm{PhD}^{4}$, Vanderlei Salvador Bagnato, $\mathrm{PhD}$, \\ Catherine Malinda Harvey, MD, and Marleny Elizabeth Martinez Gerbi, PhD ${ }^{6}$
}

\begin{abstract}
Objective: This investigation assessed the susceptibility of Streptococcus mutans and Lactobacillus acidophilus to Photodynamic Therapy (PDT) when grown simultaneously in dentine carious lesions. Background data: PDT is a technique that utilizes light to activate photosensitizers in the presence of oxygen to produce reactive radicals. Materials and methods: A culture medium of $1 \%$ glucose, $2 \%$ sucrose, $1 \%$ young primary culture of L. acidophilus $10^{8} \mathrm{CFU} / \mathrm{mL}$, and $S$. mutans $10^{8} \mathrm{CFU} / \mathrm{mL}$ was utilized to inoculate the bacterial induced caries on human dentine slabs. Different concentrations of the photosensitizer $(0.75,1.5,3.0,4.0$, and $5.0 \mathrm{~g} / \mathrm{L})$ were activated through exposure to the light-emitting diode source with a central wavelength of $450 \mathrm{~nm}$ and a fluency of $5.7 \mathrm{~J} / \mathrm{cm}^{2}$. Two light intensities $\left(19\right.$ and $\left.47.5 \mathrm{~mW} / \mathrm{cm}^{2}\right)$ were tested. Four different groups were analyzed: LD- (control group), L-D+ (drug group), L+D+1 (PDT group 1, light intensity of $19 \mathrm{~mW} / \mathrm{cm}^{2}$ ), and L+D+2 (PDT group 2, light intensity of $\left.47.5 \mathrm{~mW} / \mathrm{cm}^{2}\right)$. ANOVA/Tukey tests were utilized to compare groups $(\alpha=5 \%)$. Results: Both light intensities required $5.0 \mathrm{~g} / \mathrm{L}$ of curcumin for significant bacterial reduction $(p<0.05)$. No significant effect was found for $\mathrm{L}-\mathrm{D}+$, thus proving the absence of a potential inherent toxicity. Conclusions: Curcumin has a toxic effect on microorganisms at appreciable concentrations upon photoactivation. However, it was required to use the maximum concentration of the drug for a successful procedure.
\end{abstract}

Keywords: dentin, dentistry, photodynamic therapy

\section{Introduction}

T HE BIOFILM FORMED on the tooth surface is a microbial community embedded in a matrix of polymeric byproducts with bacterial and salivary origin. ${ }^{1,2}$ Particularly, the acidic byproducts of carbohydrate metabolism by the acidogenic microorganisms within these biofilms largely contribute to the development of dental caries. ${ }^{3,4}$

Technological advances have encouraged studies of Photodynamic Therapy (PDT) as a potential alternative to combat the formation of dental caries. PDT requires the application of a photoactive drug that can be activated upon exposure to the appropriate light source. Photon absorption leads to an electronically excited state, which in the presence of oxygen, catalyzes a cytotoxic photodynamic reac- tion that produces reactive oxygen species (ROS); these sequential oxidative reactions lead to cell death. ${ }^{5}$

A large number of studies have shown promising results for photodynamic inactivation of microorganisms related to dental caries; extensive investigations utilizing various photosensitizers (PS) and light combinations have been performed. $^{6-9}$ In addition, several publications have shown that when curcumin is utilized as PS, PDT is able to kill cariogenic bacteria, ${ }^{10-14}$ but few studies analyzed the best PDT dose-response for bacterial photodestruction in dentinal tubules using curcumin as a photosensitizer.

An ideal PS should be nontoxic and should display local toxicity only after activation by light. ${ }^{15}$ Curcumin is a dye extracted from the rhizomes of the plant Curcuma longa and effectuates antitumor, anticancer, anti-inflammatory,

\footnotetext{
${ }^{1}$ Department of Restorative Dentistry, Dental School of Pernambuco, University of Pernambuco (UPE), Camaragibe, Brazil.

${ }^{2}$ Department of Endodontics, University of Pernambuco (UPE), Camaragibe, Brazil.

${ }^{3}$ Center for Biophotonics, University of California, Davis (UCDavis), Sacramento, California.

${ }^{4}$ Department of Clinical Analysis, School of Pharmaceutical Sciences, Universidade Estadual Paulista (UNESP), Araraquara, Brazil.

${ }^{5}$ Optics Group from Physics Institute of São Carlos (IFSC), University of São Paulo (USP), São Carlos, Brazil.

${ }^{6}$ Laser Center, University of Pernambuco (UPE), Camaragibe, Brazil.

${ }^{7}$ Faculty of Dentistry, University Center Tabosa de Almeida ASCES-UNITA, Caruaru, Brazil.
} 
antioxidant, and antimicrobial medicinal activities. ${ }^{16,17} \mathrm{~A}$ number of cell culture and whole animal studies have demonstrated the essentially nontoxic effects of curcumin, which can be detected through a rather broad absorption peak in the range of 300-500 nm (maximum $430 \mathrm{~nm}$ ). ${ }^{18,19}$ Moreover, with the capability of producing high quantities at a reasonable cost, this PS could provide an economically enticing solution.

Common clinical PDT light sources include LASER, light-emitting diode (LED), and halogen lamps. LASER provides a monochromatic and highly efficient $(>90 \%)$ radiation source that can be transmitted through optical fibers in endoscopic and interstitial light-delivery devices. However, their high cost can discourage their use. However, a low priced system, such as the Diode LASER, is both convenient and reliable. This light source is limited to a single wavelength and each photosensitizer does require a separate unit due to their different absorption wavelengths. Therefore, light sources such as LED have become an attractive alternative for PDT, particularly for irradiating superficial tissue surfaces. The easy configuration of LED arrays into different irradiation geometries and their low cost provide this system with a unique advantage over LASER or diode LASER sources. Although LED does have a fixed output wavelength as well, the cost per watt is significantly lower, thus diminishing the drawback of requiring a separate unit for each photosensitizer. ${ }^{20}$

The simple PDT mechanism that activates photosensitizers such as curcumin could provide an exceptional alternative to eradicate the pernicious microorganisms that populate dental cavities. The objective of this study was to assess the photodynamic effects of curcumin activated with an LED light source (central wavelength of $450 \mathrm{~nm}$ ) on Streptococcus mutans and Lactobacillus acidophilus grown concurrently in dentine carious lesions.

\section{Materials and Methods}

\section{Dentin caries-like lesion formation}

One hundred eighty structurally normal human third molars were used, after scaling the periodontal tissue. The teeth were rigid, without the presence of caries, and there were no visible structural abnormalities. The teeth used in this study were extracted by orthodontic indication in the surgery department and Buco-Maxillofacial, Faculty of Dentistry, University of Pernambuco. A titanium trephine drill with a $4.0 \mathrm{~mm}$ internal diameter was utilized to obtain identically sized dentine slabs from the cervical third of the root. Each slab was then air-dried for $1 \mathrm{~min}$, and an average weight of $23 \mathrm{mg}$ was calculated. After an autoclave cycle at $121^{\circ} \mathrm{C}$ for $20 \mathrm{~min},{ }^{6}$ the slabs were deposited into a 24 -well plate. The wells contained $2 \mathrm{~mL}$ of a BHI (brain-heart infusion) culture medium augmented with $1 \mathrm{~g} / 100 \mathrm{~mL}$ glucose and $2 \mathrm{~g} / 100 \mathrm{~mL}$ sucrose Pro-analysis. Each $50 \mathrm{~mL}$ of medium solution was inoculated with $5 \mathrm{~mL}$ of $10^{8} \mathrm{CFU} / \mathrm{mL}$ L. acidophilus (ATCC \#ITAL-523) and $5 \mathrm{~mL}$ of $10^{8} \mathrm{CFU} /$ $\mathrm{mL}$ S. mutans (ATCC \#25175). Bacteria numbers were measured in a spectrophotometer wavelength $600 \mathrm{~nm}$ in 1$\mathrm{mL}$ cuvettes. The use of spectrophotometer with defined absorbance is the method more precise for obtaining cell numbers. The optical density was defined according to the absorption characteristics of the microorganisms. Cultures were grown until the mid-logarithmic phase. The optical density units used were (OD 600) $\sim 0.4$ and (OD 600) $\sim 0.6$ to have $\sim 10^{8}$ cells $/ \mathrm{mL}$ for $S$. mutans and L. acidophilus, respectively. The 24 -well plates were maintained in microaerobic conditions at $37^{\circ} \mathrm{C}$ for 14 days, with fresh solutions distributed every $48 \mathrm{~h}$. After the growth period, the specimens were preserved at $4^{\circ} \mathrm{C}$ until treatment.

\section{Photosensitizer}

The stock storage solution of curcumin and curcuminoids was dissolved in $N$-dimethyl-D-glucamin (PDT Pharma, Cravinhos, SP, Brazil) to a concentration of $300 \mathrm{mg} / \mathrm{mL}$, which then contained $66.7 \%$ glucamin, $17.8 \%$ curcumin, and $15.5 \%$ demethoxy and bisdemethoxycurcumin. Before the experiment began, this solution was diluted with distilled water to obtain final concentrations of $0.75,1.5,3.0$, 4.0 , and $5.0 \mathrm{~g} / \mathrm{L}$ and stored in absence of light until use.

The ultraviolet (UV)-visible absorption spectra of the stock solution were recorded by Cary 50 Bio UV-vis spectrometer (Varian, Darmstadt, Germany) using quartz cuvettes with a $1-\mathrm{cm}$ path length. These spectra were characterized by the long wavelength maximum at $430 \mathrm{~nm}$.

\section{Light source}

The system consisted of 96 LEDs (Edixeon; Edison Opto Corporation, New Taipei City, Taiwan). Each LED delivered a light intensity of 19 and $47.5 \mathrm{~mW} / \mathrm{cm}^{2}$ to the well solution ( $\sim 6 \%$ of plastic absorption of light was previously deducted), centered at a wavelength of $450 \mathrm{~nm}$, with an average fluency of $5.7 \mathrm{~J} / \mathrm{cm}^{2}$. The presence of a cooler on the sides mitigated the heating effect and allowed the system to uniformly irradiate the wells. The internal surfaces were mirrored, and the light was evenly distributed onto each well by optimizing the distance between the LEDs and the plate (Fig. 1). A power meter (Coherent, Santa Clara, CA) was utilized to assess the power density of the incident radiation.

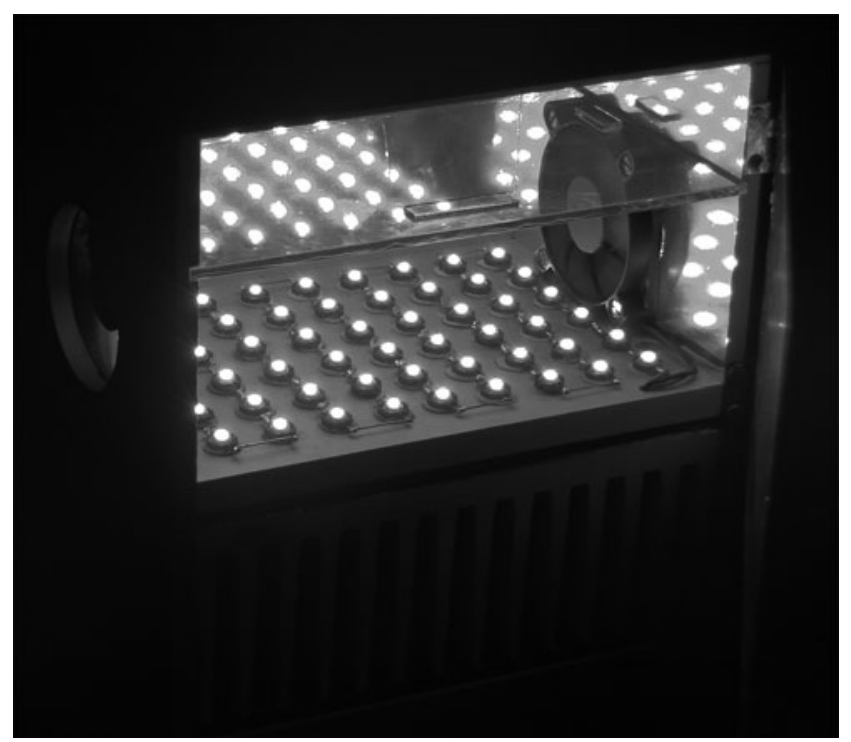

FIG. 1. Light system with 96 LEDs delivering a uniform radiation (mirrored device) with coolers to avoid heating effect. LED, light-emitting diode. 


\section{Photodynamic treatment}

The following groups were assigned: Group L-D-: no light, no drug (control group); Group L-D+: treated with curcumin (drug group); L+D+1 (PDT group 1, light intensity of $19 \mathrm{~mW} / \mathrm{cm}^{2}$ ); and $\mathrm{L}+\mathrm{D}+2$ (PDT group 2, light intensity of $47.5 \mathrm{~mW} / \mathrm{cm}^{2}$ ). The group L+D- (only light) was omitted according to previous experience of our group that observed unnoticeable variation with this level of light.

After the induction of dentine carious lesions, dentine slabs were relocated from the 24-well plates to eppendorfs containing $1 \mathrm{~mL}$ of the BHI culture medium and vortexed $(60 \mathrm{~Hz}$; Heidolph, Kelheim, Germany) for $10 \mathrm{sec}$ to eliminate unadhered bacteria. The observable biofilms on the slab surfaces were removed to expose the carious dentine tissue. Then, the slabs were deposited to the 96-well plates for PDT. Performed in triplicate, curcumin was applied to group $\mathrm{L}-\mathrm{D}+, \mathrm{L}+\mathrm{D}+1$, and $\mathrm{L}+\mathrm{D}+2(0.75,1.5,3.0,4.0$, or $5.0 \mathrm{~g} / \mathrm{L})$ for $5 \mathrm{~min}$. Groups L-D- and L-D+ were maintained at ambient temperature and covered with aluminum foil for a period equivalent to the irradiation time.

For PDT group 1, all wells were simultaneously irradiated with blue light from the diode laser for $5 \mathrm{~min}$ in the dark at room temperature. The light exposure was from below with an irradiance of $19 \mathrm{~mW} / \mathrm{cm}^{2}$ in each well and a fluence of $5.7 \mathrm{~J} / \mathrm{cm}^{2}$. For PDT group 2, the wells were irradiated for $2 \mathrm{~min}$ at $47.5 \mathrm{~mW} / \mathrm{cm}^{2}$ with a fluency of $5.7 \mathrm{~J} / \mathrm{cm}^{2}$. To avoid contamination, the plates were covered with polystyrene lids, and each 96-well plate contained only one group of three wells to avoid potential exposure from adjacent wells. In addition, the plates remained covered and undisturbed during illumination. After treatment, the carious bacterial colonies were dispersed into the medium through a forceful disturbance of the slabs in the wells. Then, the well contents were placed into an Eppendorf (the fragments were transferred with the aid of pliers and the remaining solution with Pasteur pipettes) and vortexed $(60 \mathrm{~Hz}$; Heidolph) for $60 \mathrm{sec}$. The supernatant was utilized to create serial dilutions with the BHI culture medium. Further, $100 \mu \mathrm{L}$ aliquots of these dilutions were dispensed onto blood agar plates and incubated for $72 \mathrm{~h}$ under microaerophilic conditions.
The Research and Ethics Committee of the University of Pernambuco (Protocol No. 177/08) has approved these experimental procedures with human dental specimens.

\section{Statistical analysis}

The data were processed with version 13.0 of the Statistical Package for Social Sciences (SPSS, Chicago, IL, 2006). One-way ANOVA was used to evaluate the statistical significance of the differences between mean and Tukey test to all pairwise comparisons.

\section{Results}

The data are reported as the mean of a triplicate measurement. Survival fractions in each well were calculated by counting the colonies on the plates $(\mathrm{L}+\mathrm{D}+)$ and dividing these by the number of colonies from the control group (L-D-).

The effectiveness of PDT to eradicate the $S$. mutans and L. acidophilus strains in carious dentine tissue is showed in Fig. 2. Through HSD (Honestly Significant Difference) it is demonstrated that PDT significantly reduced the number of bacteria $(p<0.05)$ in both the $\mathrm{L}+\mathrm{D}+1$ and $\mathrm{L}+\mathrm{D}+2$ groups more so than in any other group at $5.0 \mathrm{~g} / \mathrm{L}$ curcumin concentrations.

For PDT group 1 and PDT group 2, a curcumin concentration exposure of $4.0 \mathrm{~g} / \mathrm{L}$ induced a bacterial strain reduction of $44.4 \%$ and $46.4 \%$ during light exposure $(p>$ $0.05)$. Moreover, at a concentration of $5.0 \mathrm{~g} / \mathrm{L}$, a significant reduction of $69.4 \%$ and $70.4 \% \quad(p<0.05)$ was evident. Curcumin concentrations of $0.75,1.5$, and $3.0 \mathrm{~g} / \mathrm{L}$ were not sufficient enough to produce the desired toxic effect in either PDT groups. At all curcumin concentration levels, the dark assay group (L-D+) did not show toxicity, and the mean differences in the survival fractions of the drug-only group (L-D+) were low. The data are described as the mean of a triplicate measurement.

\section{Discussion}

This study demonstrated that PDT applied to artificially induced dental caries promoted a significant reduction in the viability of $L$. acidophilus and S. mutans. The use of

\section{PDT effect in dentine carious lesions}

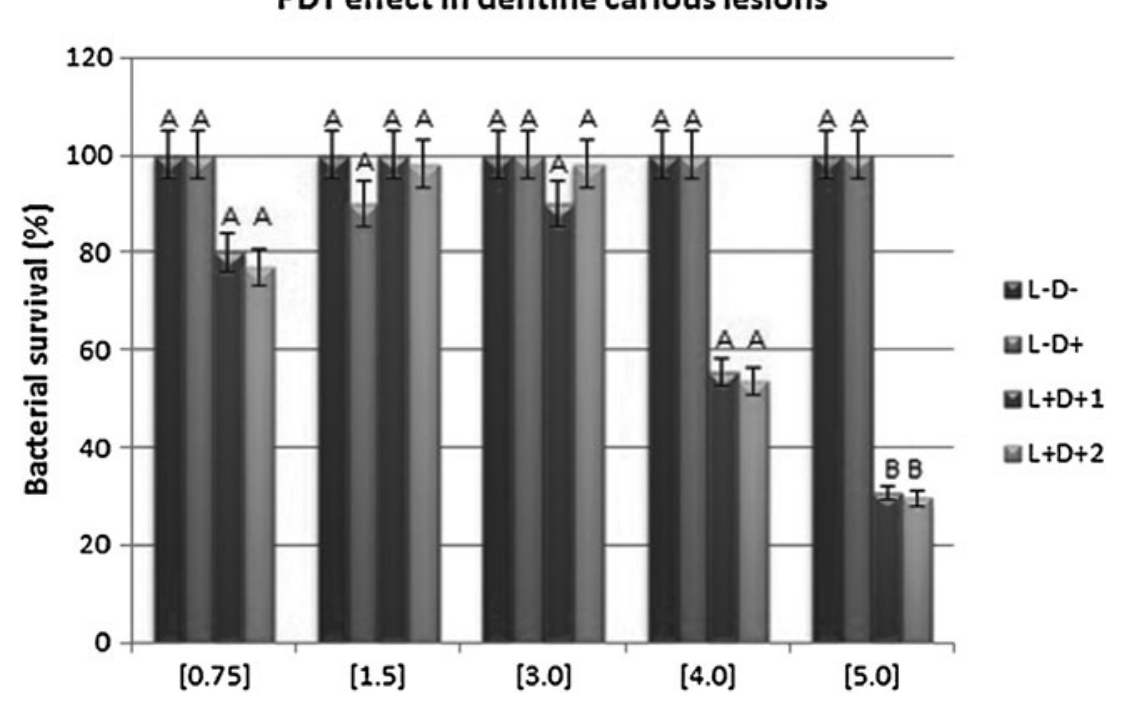

FIG. 2. Percentage of survival fractions of Streptococcus mutans and Lactobacillus acidophilus grown as multi-species in dentine carious lesions after photosensitization with different curcumin concentrations followed by irradiation with blue light at a wavelength of $450 \mathrm{~nm}$. Same letter (A) means no significant difference between the groups $(p>0.05)$. 
curcumin followed by irradiation with blue LED light reduced the viability of microorganisms. This result is in agreement with previous studies that verified the photoinactivation of cariogenic bacteria with curcumin and blue light at $450 \mathrm{~nm} .{ }^{10-14,21}$

Previously, our group utilized curcumin and an LED at $450 \mathrm{~nm}$ to eradicate oral bacteria with PDT, and a statistically significant reduction $(p<0.05)$ was noted. ${ }^{21}$ The photodynamic effects of curcumin has also been investigated in planktonic cultures and biofilm conditions. ${ }^{10,11}$ The present study indicated that PDT elicits a photoinactivation of cariogenic bacteria after the application of curcumin and an LED irradiation at $450 \mathrm{~nm}$. Moreover, the phototoxic effect seems to depend on the curcumin concentration (Fig. 2).

Some photosensitizers have a low toxicity on biofilms ${ }^{22}$ and collagen matrix, ${ }^{23}$ due to their limited accessibility into the deeper layers of microorganisms. The study of Pereira et al. ${ }^{22}$ demonstrated that lethal photosensitization mediated by methylene blue occurred predominantly in the superficial layers of the biofilms formed by Candida albicans, Staphylococcus aureus, and S. mutans. Although we have not used a methodology to identify the photosensitizer penetration depth, our results showed that under carious dentine conditions a high curcumin concentration $(5.0 \mathrm{~g} / \mathrm{L})$ was required to obtain a significant reduction in bacteria cultures.

These results suggest that the concentration of the photosensitizer significantly affects the diffusivity, especially when microorganisms are in complex culture media. The organic structure of decayed dentine was probably able to reduce the photodynamic effects by both decreasing the photosensitizer penetrability, which diminishes the binding efficiency to bacterial colonies, and attenuating the potential irradiation penetration necessary to photoactivate the dye. In vivo evaluations are needed to confirm the clinical relevance of these results, since photodynamic toxic effects are potentially influenced by the microenvironment, the presence of other biomolecules, and the other probable cellular interactions. Further studies will be developed to determine the depth at which the photosensitizer and the blue light can penetrate into the dentine and to establish effective in vivo parameters for the treatment of dentine carious lesions.

This study also evaluated the effect of different curcumin illumination times and light intensities. The PDT group 1 utilized curcumin illumination time of $5 \mathrm{~min}$ and light intensity of $19 \mathrm{~mW} / \mathrm{cm}^{2}$. For PDT group 2, curcumin illumination time of $2 \mathrm{~min}$ and light intensity of $47.5 \mathrm{~mW} / \mathrm{cm}^{2}$ were applied. A similar reduction in the feasible cells was observed for both PDT groups. Different illumination time and light intensities did not affect curcumin photodynamic effects.

da Frota et al. ${ }^{24}$ evaluated curcumin photodynamic effects on root canals contaminated with Enterococcus faecalis using two different illumination times ( 5 and $10 \mathrm{~min}$ ). In the immediate posttreatment collection, only the group that irradiated over the course of $5 \mathrm{~min}$ showed greater bacterial reduction $(p<0.05)$. Curcumin was effective with $5 \mathrm{~min}$ LED irradiation, but not with $10 \mathrm{~min}$ irradiation PDT using LED light. This result can be related to curcumin photobleaching. Dovigo et al. ${ }^{25}$ showed that irradiation fluencies larger than $5.28 \mathrm{~J} / \mathrm{cm}^{2}$ had a negligible influence on the phototoxic efficiency of curcumin. Moreover, the light ab- sorption and fluorescence of curcumin decreased as a function of illumination time, which may have been caused by photobleaching; this effectively reduces ROS production, as the authors noted after the long illumination period. ${ }^{25,26}$ Accordingly, in the present investigation, the light intensity was adjusted to the illumination time to obtain the same fluency for both PDT groups $\left(5.7 \mathrm{~J} / \mathrm{cm}^{2}\right)$.

The results showed that a curcumin illumination time of 2 or 5 min was equally effective in reducing bacteria. Evidently, only short durations of illumination are required to photoactivate the dye and induce the phototoxic effects. This represents an important parameter for clinical practice. A shorter time of clinic attendance means a lower cost treatment and higher patient satisfaction. Translational research involves a process known as translation of knowledge, in which the focus of scientific research in the area of health acquires a two-way flow of information between basic and clinical research. This results in a real applicability of knowledge and new technologies, improving clinical application of new therapeutic concepts, and, finally, providing direct benefits to the main interested in this process: patients. The aim of the translational research is to promote an interdisciplinary and two-way exchange of information from basic research findings in the laboratory to applied clinical settings involving patients and populations. In our study, the choice of curcumin as a photosensitizer allows our research to provide results that can be applied in the future of clinical practice. In dentistry, the choice of photosensitizer strongly depends upon the available light source. The PDT sources must produce adequate irradiation intensity in the activation spectrum of the photosensitizer. Commonly applied light sources in PDT are galliumaluminum-arsenide diode lasers (630-690, 830, or $906 \mathrm{~nm})$, helium-neon lasers (633 nm), and argon lasers (488-514 nm). However, the monochromatic and highly intense properties of these advantageous light sources may be outweighed by their cost. Thus, nonlaser sources, such as LED, have become an attractive alternative for PDT. Their inexpensive, flexible, and lightweight properties have made these instruments particularly conducive to the irradiation of superficial tissue surfaces. In addition, LED systems are present in common dental routine, and studies that use dental routine materials may facilitate the inclusion of PDT in dental practice without requiring the acquisition of new equipment. Therefore in this investigation, efforts were made to utilize an LED source $(450 \mathrm{~nm})$ to activate curcumin. Moreover, an effective study of an economically priced photosensitizer such as curcumin could promote the development of a PDT-type procedure into a clinical reality.

Further studies on the use of curcumin and blue light for eliminating $S$. mutans and L. acidophilus in dentine carious lesions should be encouraged. An efficient technique to sterilize the dentine tissue before a treatment is needed. PDT could deliver the solution to eliminate oral bacteria both deep in the root and away from the remaining dental tissues, and thus, contribute to the advancement of a conservative approach that effectively treats deep carious lesions.

\section{Conclusions}

These results demonstrated that PDT utilizing curcumin and blue LED light at $450 \mathrm{~nm}$ exhibited a sufficient toxicity 
against the bacterial strains of $S$. mutans and L. acidophilus grown as multi-species in dentine carious lesions. This study suggests that this technique may prove to proficiently treat caries-related diseases.

\section{Acknowledgments}

Funding for this research was provided by the Research Support Foundation of São Paulo State (FAPESP) and by the Science and Technology Support Foundation of Pernambuco State (FACEPE).

\section{Author Disclosure Statement}

No competing financial interests exist.

\section{References}

1. Marsh PD. Microbiology of dental plaque biofilms and their role in oral health and caries. Dent Clin North Am 2010;54:441-454.

2. Davey ME, O'Toole GA. Microbial biofilms: from ecology to molecular genetics. Microbiol Mol Biol Rev 2000;64: 847-867.

3. Svensäter G, Borgström M, Bowden GH, Edwardsson S. The acid-tolerant microbiota associated with plaque from initial caries and healthy tooth surfaces. Caries Res 2003; 37:395-403.

4. Selwitz RH, Ismail AI, Pitts NB. Dental caries. Lancet 2007;369:51-59.

5. Al-Watban FA, Zhang XY. Photodynamic therapy of human undifferentiated thyroid carcinoma-bearing nude mice using topical 5-aminolevulinic acid. Photomed Laser Surg 2005;23:206-211.

6. Giusti JS, Santos-Pinto L, Pizzolito AC, et al. Antimicrobial photodynamic action on dentin using a lightemitting diode light source. Photomed Laser Surg 2008; 26:281-287.

7. Lima JP, Sampaio de Melo MA, Borges FM, et al. Evaluation of the antimicrobial effect of photodynamic antimicrobial therapy in an in situ model of dentine caries. Eur J Oral Sci 2009; 117:568-574.

8. Melo MAS, de-Paula DM, Lima JPM, et al. In vitro photodynamic antimicrobial chemotherapy in dentin contaminated by cariogenic bacteria. Laser Phys 2010;20: 1504-1513.

9. Monteiro-Oliveira MP, Rodrigues LKA, Melo MAS, Nobre-dos-Santos M. Photodynamic therapy effect in carious bovine dentin - an in vitro study. J Oral Laser Applications 2010;10:29-36.

10. Araújo NC, Fontana CR, Bagnato VS, Gerbi ME. Photodynamic effects of curcumin against cariogenic pathogens. Photomed Laser Surg 2012;30:393-399.

11. Araújo NC, Fontana CR, Bagnato VS, Gerbi ME. Photodynamic antimicrobial therapy of curcumin in biofilms and carious dentine. Lasers Med Sci 2014;29:629-635.

12. Paschoal MA, Lin M, Santos-Pinto L, Duarte S. Photodynamic antimicrobial chemotherapy on Streptococcus mutans using curcumin and toluidine blue activated by a novel LED device. Lasers Med Sci 2015;30:885-890.

13. Paschoal MA, Santos-Pinto L, Lin M, Duarte S. Streptococcus mutans photoinactivation by combination of short exposure of a broad-spectrum visible light and low concentrations of photosensitizers. Photomed Laser Surg 2014; 32:175-180.
14. Tonon CC, Paschoal MA, Correia M, et al. Comparative effects of photodynamic therapy mediated by curcumin on standard and clinical isolate of Streptococcus mutans. J Contemp Dent Pract 2015;16:1-6.

15. Meisel P, Kocher T. Photodynamic therapy for periodontal diseases: state of the art. J Photochem Photobiol B 2005; 79:159-170.

16. Aggarwal BB, Sundaram C, Malani N, Ichikawa H. Curcumin: the Indian solid gold. Adv Exp Med Biol 2007; 595:1-75.

17. Haukvik T, Bruzell E, Kristensen S, Tønnesen HH. Photokilling of bacteria by curcumin in selected polyethylene glycol 400 (PEG 400) preparations. Studies on curcumin and curcuminoids, XLI. Pharmazie 2010;65:600-606.

18. Priyadarsini KI. Photophysics, photochemistry and photobiology of curcumin: studies from organic solutions, biomimetics and living cells. J Photochem Photobiol C 2009; 10:81-95.

19. Haukvik T, Bruzell E, Kristensen S, Tønnesen, HH. A screening of curcumin derivatives for antibacterial phototoxic effects studies on curcumin and curcuminoids. XLIII. Pharmazie 2011;66:69-74.

20. Wilson BC, Patterson MS. The physics, biophysics and technology of photodynamic therapy. Phys Med Biol 2008; 53:61-109.

21. Araújo NC, Fontana CR, Bagnato VS, Gerbi ME. Overallmouth disinfection by photodynamic therapy using curcumin. Photomed Laser Surg 2012;30:96-101.

22. Pereira CA, Romeiro RL, Costa AC, Machado AK, Junqueira JC, Jorge AO. Susceptibility of Candida albicans, Staphylococcus aureus, and Streptococcus mutans biofilms to photodynamic inactivation: an in vitro study. Lasers Med Sci 2010;26:341-348.

23. Williams JA, Pearson GJ, Colles MJ, Wilson M. The photoactivated antibacterial action of toluidine blue $\mathrm{O}$ in a collagen matrix and in carious dentine. Caries Res 2004;38: 530-536.

24. da Frota MF, Guerreiro-Tanomaru JM, Tanomaru-Filho M, Bagnato VS, Espir CG, Berbert FL. Photodynamic therapy in root canals contaminated with Enterococcus faecalis using curcumin as photosensitizer. Lasers Med Sci 2015;30: 1867-1872.

25. Dovigo LN, Pavarina AC, Ribeiro AP, et al. Investigation of the photodynamic effects of curcumin against Candida albicans. Photochem Photobiol 2011;87:895-903.

26. Dovigo LN, Pavarina AC, Carmello JC, Machado AL, Brunetti IL, Bagnato VS. Susceptibility of clinical isolates of Candida to photodynamic effects of curcumin. Lasers Surg Med 2011;43:927-934.

Address correspondence to: Natália Costa Araújo Department of Restorative Dentistry Dental School of Pernambuco University of Pernambuco 1650 Gal Newton Cavalcanti Avenue Camaragibe 54753-020

Brazil

E-mail: nataliacosta84@yahoo.com.br

Received: April 29, 2016. Accepted after revision: October 13, 2016. Published online: January 12, 2017. 